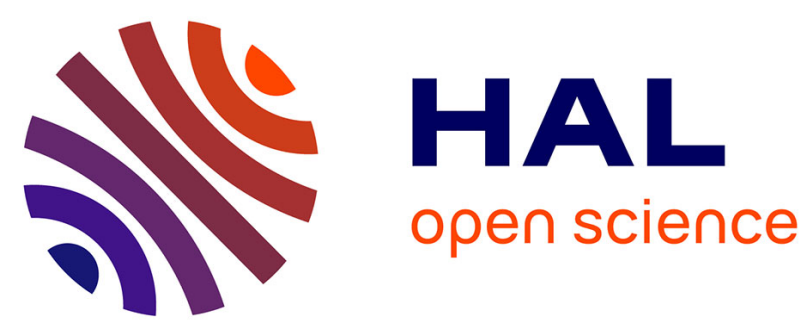

\title{
PHOTOLUMINESCENCE AND ESR STUDIES OF LOCALIZED STATES IN AMORPHOUS PHOSPHORUS
}

\author{
B. Shanabrook, S. Bishop, P. Taylor
}

\section{- To cite this version:}

B. Shanabrook, S. Bishop, P. Taylor. PHOTOLUMINESCENCE AND ESR STUDIES OF LOCALIZED STATES IN AMORPHOUS PHOSPHORUS. Journal de Physique Colloques, 1981, 42 (C4), pp.C4-865-C4-868. 10.1051/jphyscol:19814189 • jpa-00220815

HAL Id: jpa-00220815 https://hal.science/jpa-00220815

Submitted on 1 Jan 1981

HAL is a multi-disciplinary open access archive for the deposit and dissemination of scientific research documents, whether they are published or not. The documents may come from teaching and research institutions in France or abroad, or from public or private research centers.
L'archive ouverte pluridisciplinaire HAL, est destinée au dépôt et à la diffusion de documents scientifiques de niveau recherche, publiés ou non, émanant des établissements d'enseignement et de recherche français ou étrangers, des laboratoires publics ou privés. 
JOURNAL DE PHYSIQUE

Colloque C4, supplément au no10, Tome 42, octobre 1981

page $\quad \mathrm{C}_{4}-865$

\title{
PHOTOLUMINESCENCE AND ESR STUDIES OF LOCALIZED STATES IN AMORPHOUS PHOSPHORUS
}

\author{
B.V. Shanabrook, S.G. Bishop and P.C. Taylor \\ Naval Research Laboratory, Washington, D.C. 20375, U.S.A.
}

\begin{abstract}
Photoluminescence and electron spin resonance measurements have been performed in bulk a-red $P$. We observe a photoluminescence band at $1.40 \mathrm{eV}$ which exhibits a sensitivity to the excitation energy employed. Specifically, the peak of this band progresses to lower energies for higher energy excitation. Electron spin resonance measurements indicate $\sim 10^{17} \mathrm{spins} / \mathrm{cm}^{3}$ for "cold dark" and optically induced (6328 $)$ conditions. In contrast to similar measurements in bulk a-As, thermally generated paramagnetism is not apparent in bulk a-red $P$ up to $300 \mathrm{~K}$. These results are compared with various defect models proposed for the pnictides.
\end{abstract}

Introduction,- Photoluminescence (PL) and electron spin resonance (ESR) measurements in amorphous arsenic (a-As) have been interpreted in terms of localized electronic defect states such as two- or four-fold coordinated pnictide atoms $(1,2,3)$ or "vacancies" $(4,5)$ in the normally three-fold coordinated pnictide network. Semiconducting ( $E=2.1 \mathrm{eV}(6)$ ) bulk amorphous $(\mathrm{a}-)$ red $P$ exhibits an average bond angle of $102^{\circ}$, which is significantly larger than the $98^{\circ}$ bond angle characteristic of a-As (1). This difference suggests that bulk a-red $P$ may provide a useful additional test of the various defect models proposed for a-As. We have performed photoluminescence (PL), PL excitation and ESR measurements on bulk a-red P, to further elucidate the nature of defects in the pnictides.

Experimental.- PL and PL excitation measurements were performed at $4.2 \mathrm{~K}$ with a SPEX 1701 monochromator. An appropriate choice of filters prevented scattered excitation light from being detected by either the S-1 photomultiplier or PbS detector. A krypton laser and tungsten lamp were employed as excitation sources. The ESR spectra were obtained with a standard X-band ( $9 \mathrm{GHz}$ ) spectrometer with variable temperature capability and an optical access cavity. Samples of bulk a-red $p$ were obtained from Mining and Chemical Products. The preparation procedure has been described elsewhere (7).

Results.- Shown in Fig. 1 are photoluminescence spectra of bulk a-red $P$ obtained with the excitation energies indicated. Luminescence energies above $1.24 \mathrm{eV}$ were detected with an S-1 photomultiplier, while lower energies were observed with a cooled PbS detector. Excitation with $2.60 \mathrm{eV}$ and $2.38 \mathrm{eV}$ radiation resulted in very weak luminescence signals which could not be observed with the $\mathrm{PbS}$ detector. In contrast to that which has previously been observed in the chalcogenide glasses ( 8 ), both the position and width of the luminescence band in bulk a-red $P$ exhibit subtle changes with excitation energy. These changes are further documented in Table I where both the peak position and full width at half maximum are shown for various excitation energies. The peak of the luminescence band progresses, although not monotonically, to higher energies with lower energy excitation. The position of the luminescence band in bulk a-As also exhibits a sensitivity to excitation energy ( 9 ). Previous luminescence measurements (10) on bulk a-red $P$ are in substantial agreement with those presented here. The previous measurements, which were not corrected for the throughput of the spectrometer, found a narrower half width for the luminescence band of $0.36 \mathrm{eV}$. 


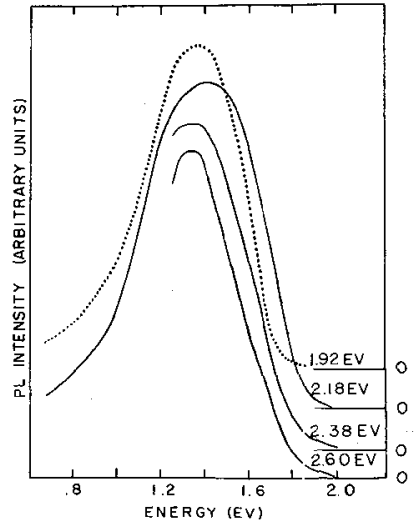

\begin{tabular}{ccc} 
Energy $(\mathrm{eV})$ & Peak Position $(\mathrm{eV})$ & Width $(\mathrm{eV})$ \\
\hline 1.92 & 1.39 & .52 \\
2.18 & 1.44 & .62 \\
2.38 & 1.38 & - \\
2.60 & 1.35 & -
\end{tabular}

Fig. 1. PL spectra of bulk a-red $P$ obtained with four different excitation energies. The zero intensity levels are indicated by horizontal lines at the right of the figure.

PL excitation (PLE) spectra for bulk a-red $P$ are shown in Fig. 2. The PL excitation of those portions of the luminescence band centered at $1.65 \mathrm{eV}$ and $1.375 \mathrm{eV}$ are shown in Fig. 2(a) while the excitation of luminescence lower in energy than $1.13 \mathrm{eV}$ and $.67 \mathrm{eV}$ are shown in Fig. 2(b). These PLE spectra consist of a single peak with the typical high energy fall-off observed in amorphous semiconductors (8), while the lower energy edge indicates a rapid rise at $\sim 1.9 \mathrm{eV}$. Recent optical studies (6) of thin films of sputtered a-P indicate that the absorptign coefficient is rapidly rising in this regime due to band-to-band absorption $\left(\alpha \sim 10^{3} \mathrm{~cm}^{-1}\right.$ at $2.0 \mathrm{eV}$ ). This fact clearly shows that the PL band (peak at $\sim 1.4 \mathrm{eV}$ ) occurs substantially below the optical absorption edge. The spectra shown in Fig. 2 are to be contrasted with the twin peaked PL excitation spectrum of bulk a-As (9). In a-As, the lower energy peak occurs significantly below the band edge and suggests that below gap states are involved in the PL excitation. Although the twin peaked excitation spectrum in bulk a-As is not observed in bulk a-red $P$, the low energy portion of the luminescence band $(<1.13 \mathrm{eV})$ can be excited by energies between 1.5 and $1.8 \mathrm{eV}$ (see Fig. 2(b)). This situation suggests, as in bulk a-As, that below-gap absorption states are involved in the production of photoluminescence. However, the concentration of these states appears to be smaller in bulk a-red $P$ than in bulk a-As.

Electron spin resonance studies have proved useful for the investigation of localized electronic states and defects in the chalcogenide glasses (8) as well as a-As (4). The ESR spectra of bulk a-red $P$ at $4.2 \mathrm{~K}$ and $100 \mathrm{~K}$ are shown in Fig. 3 . Excessive microwave saturation necessitated the use of the dispersion mode for the ESR measurements at temperatures less than 50K. Under certain circumstances the derivative of the high power dispersion mode can approximate an absorption spectrum. At $4.2 \mathrm{~K}$ the linewidth of the pseudo absorption spectrum of Fig. 3(a) is 280 Gauss. A similar width is extracted from the usual derivative of the absorption spectrum at temperatures above $\sim 50 \mathrm{~K}$ ( $\mathrm{Fig} .3(\mathrm{~b})$ ). The intensity of this ESR line increases after the sample has been irradiated with $6328 \AA$ radiation at $4.2 \mathrm{~K}$. The comparison of
this line with a standard pitch sample yields an estimated spin density of $\sim 10$ cm for both the cold dark and optically induced resonances. Similar concentrations have been observed in a-As under comparable conditions. However, Fig. 4 indicates 

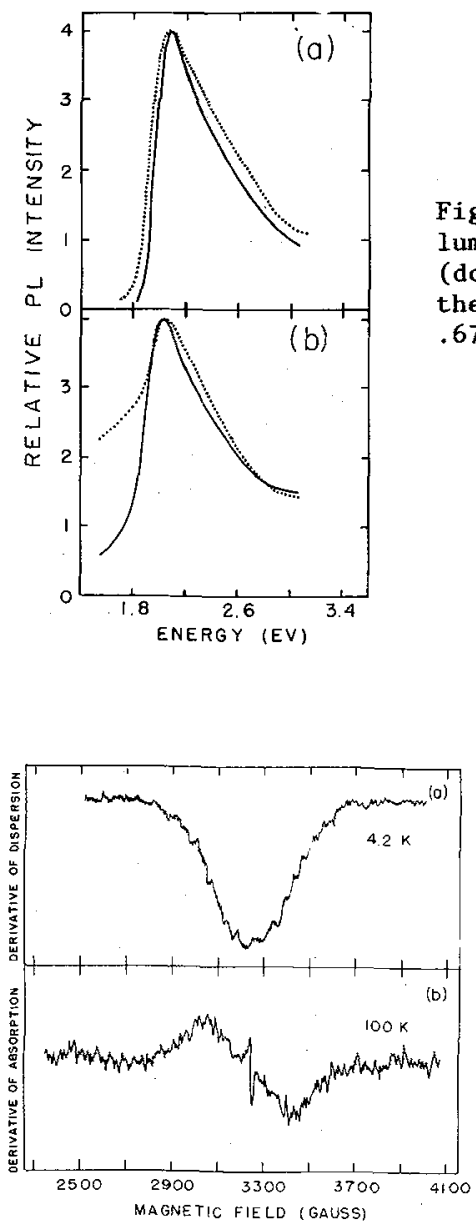

Fig. 3. Typical ESR spectra of bulk $a-r e d ~ P$ at (a) $4.2 \mathrm{~K}$ and (b) $100 \mathrm{~K}$.
Fig. 2. (a) Bulk a-red P PL excitation spectra of luminescence occurring at $1.65 \mathrm{eV}$ (solid) and $1.375 \mathrm{eV}$ (dotted). (b) PL excitation spectra of portions of the PL band with energies less than $1.13 \mathrm{eV}$ (solid) and $.67 \mathrm{eV}$ (dotted).

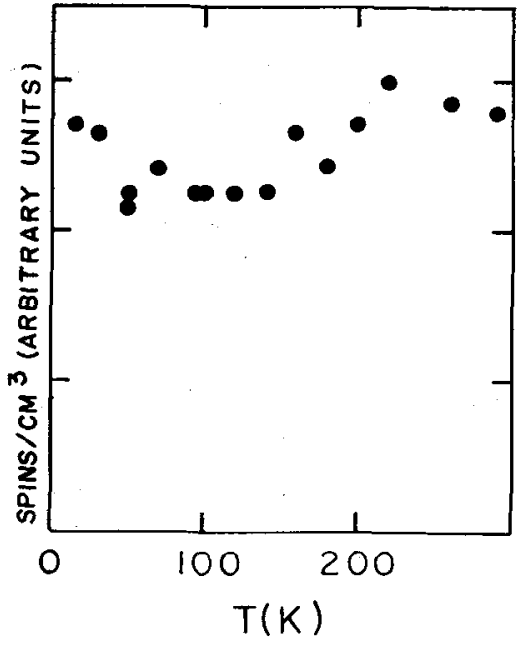

Fig. 4. The temperature dependence of the spin concentrations in bulk a-redP.

that the thermally generated paramagnetism observed in amorphous As (4) is not apparent in bulk a-red $P$ up to $300 K$. These data exhibit significant scatter above $120 \mathrm{~K}$ due to difficulties in subtracting out the growth of a new line which is due to an impurity species which is probably iron. Nevertheless, one observes that the concentration of spins up to $300 \mathrm{~K}$ is essentially constant. These data indicate that if thermally generated paramagnetism exists in bulk a-red $P$, then the activation energy must be larger than 25 meV.

As one might expect, an analysis of the s-p hybridization (4) of the wave function of the unpaired spin indicates that there is greater hybridization in the case of bulk a-red $P$ than in a-As. If one assumes that the unpaired spin is localized on a single $P$ atom, then the observed ESR linewidth indicates that there is $\sim 8-10 \%$ s-admixture to the predominantly p-type wave function for the unpaired spin. This hybridization is compared with $\sim 5 \%$ s admixture in a-As (4).

Discussion.- We have observed a PL band in bulk a-red $P$ at $\sim 1.40 \mathrm{eV}$ which occurs $.65 \mathrm{eV}$ below the peak in the excitation spectrum. The approximate midgap position 
as well as the rather large width of the PL band suggest that strong electronphonon interactions exist at the PL site. The standard semi-classical treatment (8) for such a situation yields, $\sigma=1.66$ (2Whw) $)^{\frac{2}{2}}$, where $\sigma$ is the full width of the PL band at half maximum, $W$ is the distortion energy and hw is the phonon energy.

Although it is not possible to determine the value of $2 W$, it is certainly less than $.65 \mathrm{eV}$. Assuming a phonon frequency of $.047 \mathrm{eV}$ (7), in addition to the upper limit of $.65 \mathrm{eV}$ for $2 \mathrm{~W}$, the half width of the PL band is predicted to be .29 $\mathrm{eV}$. This estimate is to be contrasted with the observed width of $\sim .57 \mathrm{eV}$. The substantial difference between the observed and calculated PL band width may arise as a consequence of two different mechanisms. It is possible (a) that more than one type of defect is contributing to the PL band at $1.4 \mathrm{eV}$. This suggestion is consistent with the subtle changes observed in the PL band as a function of excitation energy. However, it is noted that the PL band does not exhibit features (such as two separate peaks or even inflections) arising from two or more types of defects. In addition, (b) the energy of a particular type of defect may vary from site to site in an amorphous network. Such an occurrence would provide an additional broadening of both the PL and absorption bands. The PL band arising from such a distribution of defect sites would be expected to exhibit sensitivity to excitation energy, as is observed experimentally.

Some authors $(1,2,3)$ have suggested that the large admixture of $s$ and $p$ wave functions in bulk a-red $P$ would result in large negative effective correlation energies. Specifically, these authors $(1,2)$ have attributed the thermally generated paramagnetism in bulk a-As to the conversion of positively charged four-fold and negatively charged two-fold coordinated defects into neutral paramagnetic two-fold coordinated As atoms. Because the $s-p$ hybridization is expected to be greater in bulk a-red $P$ than in a-As these authors predicted that a much smaller paramagnetic signal would be observed in bulk a-red $P$. In contrast, we report the observation of an ESR signal in the absence of illumination which is comparable to that observed in a-As. The wave functions for these spins in bulk a-red $P$ exhibit large amounts of $p$ bonding character $(\sim 90 \%)$ and a positive effective electronic correlation energy.

Other authors $(4,5)$ have proposed that the excitation of electrons from weakly bonded states at vacancies in the threefold coordinated As network yields the observed thermally generated paramagnetism. The fact that there is no thermally induced paramagnetism in bulk a-red $\mathrm{P}$ below $300 \mathrm{~K}$ even though there is an ESR response at $4.2 \mathrm{~K}$ which is equivalent in magnitude to that oserved in a-As $\left(\sim 10^{17} \mathrm{~cm}^{-3}\right)$ is consistent with this interpretation. One requires that there are fewer vacancies in bulk a-red $P$ than in a-As and that these vacancies are all paramagnetic at 4.2K.

\section{References}

1. GREAVES G. N., ElliotT S. R. and DAVIS E. A., Adv. Phys. 28 (1978) 49.

2. ELLIOTT S. R. and DAVIS E. A., J. Phys. C 12 (1979) 2577.

3. KASTNER M. and FRITZSCHE H., Phil. Mag. B $\frac{37}{37}$ (1978) 199.

4. TAYLOR P. C., FRIEBELE E. J. and BISHOP S. G., Solid State Commun. 28 (1978) 247.

5. POLLARD W. B. and JOANNOPOULOS J. D., Phys. Rev. B 19 (1979) 4217.

6. PILIONE L. J., POMIAN R. J., LANNIN J. S. SSC (to be published). Note: The value of $2.1 \mathrm{eV}$ was obtained from a sputtered $\mathrm{P}$ film exhibiting similar structural and vibrational properties as the bulk material.

7. SHANABROOK B. V. and LANNIN J. S., Proc. 14th Int. Conf. of Physics of Semiconductors, 1978, p. 713.

8. STREET R. A., Adv. Phys. 25 (1977) 397.

9. KIRBY P. B. and DAVIS E. A., Proc. 14th Int. Conf. of Physics of Semiconductors, 1978 , p. 1309.

10. KIRBY P. B. and DAVIS E. A., 8th Int. Conf. on Amrp. and Liquid Semiconductors, 1980, p. 945 . 